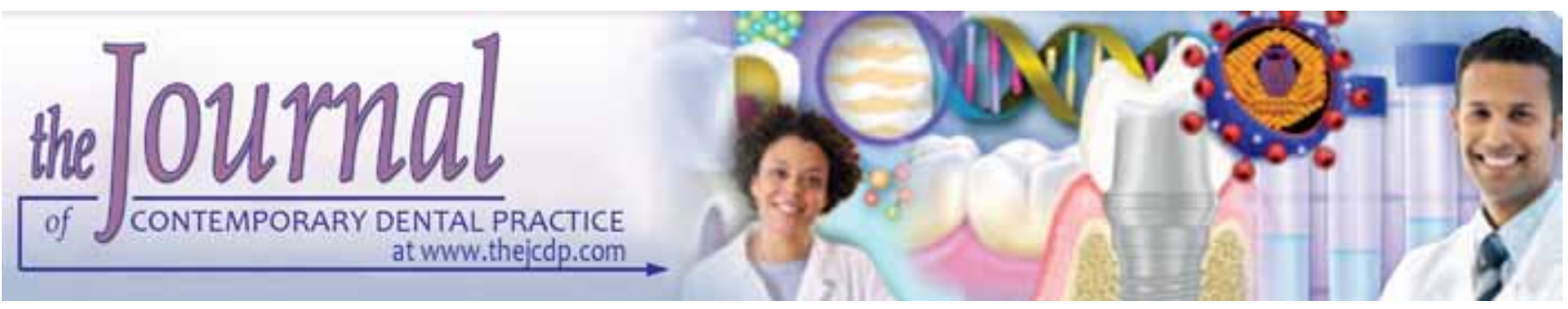

\title{
The Role of Transcutaneous Electrical Nerve Stimulation in the Management of Temporomandibular Joint Disorder
}

\author{
${ }^{1}$ Kamran Habib Awan, ${ }^{2}$ Shankargouda Patil
}

\begin{abstract}
Temporomandibular joint disorders (TMD) constitutes of a group of diseases that functionally affect the masticatory system, including the muscles of mastication and temporomandibular joint (TMJ). A number of etiologies with specific treatment have been identified, including the transcutaneous electrical nerve stimulation (TENS). The current paper presents a literature review on the use of TENS in the management of TMD patients.

Temporomandibular joint disorder is very common disorder with approximately $75 \%$ of people showing some signs, while more than quarter (33\%) having at least one symptom. An attempt to treat the pain should be made whenever possible. However, in cases with no defined etiology, starting with less intrusive and reversible techniques is prescribed. Transcutaneous electrical nerve stimulation is one such treatment modality, i.e. useful in the management of TMD. It comprises of controlled exposure of electrical current to the surface of skin, causing hyperactive muscles relaxation and decrease pain.

Although the value of TENS to manage chronic pain in TMD patients is still controversial, its role in utilization for masticatory muscle pain is significant. However, an accurate diagnosis is essential to minimize its insufficient use. Well-controlled randomized trials are needed to determine the utilization of TENS in the management of TMD patients.
\end{abstract}

Keywords: Masticatory muscles, Orofacial pain, Review, Temporomandibular disorder, Transcutaneous electrical nerve stimulation.

How to cite this article: Awan $\mathrm{KH}$, Patil S. The Role of Transcutaneous Electrical Nerve Stimulation in the Management

\footnotetext{
${ }^{1}$ Department of Oral Medicine and Diagnostic Sciences, College of Dentistry, King Saud University, Riyadh, Saudi Arabia

${ }^{2}$ College of Dentistry, Jazan University, Jazan, Saudi Arabia

Corresponding Author: Kamran Habib Awan, Assistant Professor, Department of Oral Medicine and Diagnostic Sciences, College of Dentistry, King Saud University, Riyadh, Saudi Arabia, Phone: (966) (1) 467-7422, e-mail: kamranhabibawan@ gmail.com
}

of Temporomandibular Joint Disorder. J Contemp Dent Pract 2015;16(12):984-986.

Source of support: Nil

Conflict of interest: None declared

\section{INTRODUCTION}

Temporomandibular joint disorders (TMD) constitutes of a group of diseases or disorders that mainly affect the masticatory system, particularly the temporomandibular joint (TMJ) and related structures. ${ }^{1}$ Majority of the people (approximately 75\%) have been reported to have TMD signs and symptoms, with more than quarter $(33 \%)$ having at least one symptom. ${ }^{2}$ However, only a fraction of population has serious enough problem to seek clinical treatment.

Temporomandibular joint disorder patients present with a number of signs and symptoms, including pain, malocclusion, altered joint function with or without deviation, clicking and/or restricted movement. ${ }^{3}$ In addition, these patients may suffer from headache, tinnitus, altered vision, and other neurologic sings and symptoms might likewise go with TMD. ${ }^{4}$ Patients with chronic TMD have also reported to present with signs and symptoms of depression, insomnia and low self-confidence. ${ }^{5}$

The etiology of TMD is multifactorial with no special or individual source. Factors that may initiate or disseminate the disorder include malocclusion, muscle hyperactivity, trauma, emotional stress. ${ }^{6}$ Factors related to occlusion have been widely researched in recent years leading to increased use of occlusal splints and orthodontic treatment. ${ }^{7}$ However, epidemiological data and systematic reviews have found poor or inexistent association of occlusion and TMD is poor or inexistent. ${ }^{7}$

An acceptable TMD classification based on different signs and symptoms is necessary to achieve an accurate diagnosis. A number of TMD classifications have been proposed and used, the classifications by the American 
Academy of Orofacial Pain (AAOP) and Research Diagnostic Criteria for Temporomandibular Disorders (RDC/TMD) are the ones that are widely used. ${ }^{8}$

Various treatment modalities have shown to be effective in reducing pain and restoring function of the masticatory system. ${ }^{9}$ Transcutaneous electrical nerve stimulation (TENS) is one such treatment modality that merits unique consideration. Transcutaneous electrical nerve stimulation is not only safe to use but also result in reduced pain and electromyographic (EMG) activity of muscles of mastication. There are a few studies on the effectiveness of TENS therapy. ${ }^{10-13}$ This study is a literature review on the role of TENS in the management of TMD.

\section{MATERIALS AND METHODS}

To find relevant and adequate articles for the literature review, a detailed automated search of Cochrane, MEDLINE and PubMed databases was conducted from Jan 1, 1966, through March, 17, 2014. A combination of the following keywords was used: 'Temporomandibular disorder', 'trigger points', 'TENS', 'transcutaneous electrical nerve stimulation', and 'orofacial pain'. Review articles were also searched for additional articles missed in the automated searches. Experimental studies, review articles, letters to the editor, unpublished data and articles not published in English were excluded.

\section{DISCUSSION}

Transcutaneous electrical nerve stimulation therapy is fundamentally the utilization of a device managing low voltage, pulsed electrical current as a two-phase, symmetric or asymmetric wave, adjusted as a positive square semi-wave and a negative peak. Electrodes are connected to the skin surface aimed to unwind hyperactive muscles and alleviate pain. ${ }^{14}$ A number of TENS devices are available in the market, however, there is a lack of standardization mechanism.

Transcutaneous electrical nerve stimulation is a cost-effective, safe and noninvasive treatment modality for a number of painful conditions. ${ }^{15}$ Electrodes may be silicone based that either gel application mechanism or self-adhesive. They are placed at the origin of pain or close to the site of highest pain, within the same dermatome, myotome and/or myofacial trigger points. They can also be placed on the pathway of peripheral nerves to further reduce pain and/or maintain the pain-free setting.

Transcutaneous electrical nerve stimulation devices are classified into two groups on the basis of pulse frequencies: high frequency $(>50 \mathrm{~Hz})$ and low frequency $(<10 \mathrm{~Hz})$. Devices that are used in the dental settings are generally mixed, with both low and high frequency currents. High frequency $(50-150 \mathrm{~Hz})$ devices have low intensity and have more centralized mechanism of action aimed at relieving long-lasting pain. Devices that have low frequency are generally specified for muscle relaxation as they essentially have peripheral mechanism of action. Intensity of the devices can be adjusted according to patients' sensitivity, to avoid muscle contractions and to achieve hypoesthesia or paresthesia of the affected area. Studies have shown intensities of 10 to 30 milliamps to be appropriate, resulting in few fasciculations. Further, pulse times between 40 and 75 microseconds are found to be effective.

In dentistry, TENS is mainly used to manage longlasting pain in certain cases and relax masticatory muscles. ${ }^{16,17}$ Studies have shown that at rest, patients suffering from muscular TMD have higher myoelectric activity of jaw elevator muscles than control group. ${ }^{10} \mathrm{It}$ is hypothesized that this increase myoelectric activity is due to sensory-motor interactions of craniofacial segment, resulting in modification in action potentials generations and, ultimately, myoelectric amplitude. Transcutaneous electrical nerve stimulation has been used to reduce the myoelectric activity in the anterior portion of these muscles along with pain relief.

The useful effects of TENS are explained by different theories. One theory is that TENS directly stimulates the motor nerves resulting in rhythmic contractions of the masticatory muscles. This causes increased blood flow and oxygen to the muscles, and decreased interstitial edema and build up of harmful toxins. The overall result is decreased pain and fatigue of masticatory muscles. ${ }^{18}$

Another theory, called the gate of pain theory was presented in 1965 to explain the electroanalgesia. ${ }^{18}$ According to the theory, dorsal horn of the spinal cord contains a gate that controls the nociceptive entrance through small diameter afferent nerves. Tactile or pressure stimulation through electric impulses on large diameter peripheral afferents may result in inhibition of sensory neuron stimulations to spinal and supraspinal structures, effectively closing the gate. So, TENS would have both central and peripheral mechanisms of action. ${ }^{19}$

Transcutaneous electrical nerve stimulation have been reported to be effective as a treatment modality for TMD, both individually or in combination with other modalities. A pilot study was conducted to evaluate the electromyographic biofeedback and TENS in patients suffering from bruxism. ${ }^{20}$ The authors concluded that although both therapies resulted in relaxation of muscles of mastication, TENS was more effective in decreasing the electromyographic activity of the masseter muscle. Another study reported that TENS together with interocclusion devices (IOD) resulted in a new spatial position of the jaw. ${ }^{21}$ This new occlusion significantly 
improved the bite quality and strength, increased the efficiency of most evaluated muscle $(p<0.05)$ and reduced the pain.

In a study, TENS was used in combination with occlusal splints and TENS to treat patients suffering from TMD and bruxism. ${ }^{22}$ The authors concluded that both treatment modalities were insignificant in improving signs and symptoms in TMD patients. However, other studies have reported usefulness of occlusal splints in bruxism ${ }^{23}$ and non-bruxism ${ }^{24}$ patients. In one study, authors assessed the influence of TENS combined with occlusal splint and physical therapy on craniomandibular disorders. They concluded that majority of the patients $(83 \%)$ showed a significant decrease in signs and symptoms of craniomandibular disorders following 6 weeks of use of the treatment modalities.

\section{CONCLUSION}

Transcutaneous electrical nerve stimulation is an alternate treatment option for patients suffering from TMD. However, inconsistent nature of TMD patients necessitates randomized controlled studies in specific populations to categorize patients and diseases response. Furthermore, it is also important to identify facial pain syndromes that may benefit from adjuvant TENS therapy. At present, the utilization of TENS for the most part relies on upon clinical experience and its handling by health professionals.

\section{REFERENCES}

1. Okeson JP, de Leeuw R. Differential diagnosis of temporomandibular disorders and other orofacial pain disorders. Dent Clin North Am 2011;55(1):105-120.

2. Yap AU, Ho VC. Temporomandibular disorders: an overview. Singapore Med J 1999;40(3):179-182.

3. Clark GT, Seligman DA, Solberg WK, et al. Guidelines for the examination and diagnosis of temporomandibular disorders. J Craniomandib Disord Facial Oral Pain 1989;3(1):7-14.

4. Fricton JR. Temporomandibular muscle and joint disorders. Pain: Clinical Updates 2004;12(2):1-6.

5. McNeely ML, Armijo Olivo S, Magee DJ. A systematic review of the effectiveness of physical therapy interventions for temporomandibular disorders. Phys Ther 2006;86(5):710-725.

6. Januzzi E, Alves BMF, Grossmann E, et al. Occlusion and temporomandibular disorders: a critical analysis of the literature. Rev Dor 2010;11(4):329-333.

7. Koh H, Robinson PG. Occlusal adjustment for treating and preventing temporomandibular joint disorders. J Oral Rehabil 2004;31(4):287-292.
8. Dworkin SF. Research diagnostic criteria for Temporomandibular disorders: current status and future relevance. J Oral Rehabil 2010;37(10):734-743.

9. ParkerMW.Adynamicmodel of etiology in temporomandibular disorders. J Am Dent Assoc 1990;120(3):283-290.

10. Rodrigues D, Oliveira AS, Bérzin F. Effect of conventional TENS on pain and electromyographic activity of masticatory muscles in TMD patients. Braz J Oral Sci 2004;18(4): 290-295.

11. Kamyszek G, Ketcham R, Garcia R Jr, et al. Electromyographic evidence of reduced muscle activity when ULF-TENS is applied to the Vth and VIIth cranial nerves. Cranio 2001;19(3): 162-168.

12. Treacy K. Awareness/relaxation training and transcutaneous electrical neural stimulation in the treatment of bruxism. J Oral Rehabil 1999;26(4):280-287.

13. Cooper BC. The role of bioelectronic instrumentation in the documentation and management of Temporomandibular disorders. Oral Surg Oral Med Oral Pathol Oral Radiol Endod 1997;83(1):91-100.

14. Esposito CJ, Shay JS, Morgan B. Electronic dental anesthesia: a pilot study. Quintessence Int 1993;24(3):167-170.

15. Esposito CJ, Shay JS, Morgan B. Electronic dental anesthesia: a pilot study. Quintessence Int 1993;24(3):167-170.

16. Walsh DM, Howe TE, Johnson MI, et al. Transcutaneous electrical nerve stimulation for acute pain. Cochrane Database of Systematic Reviews 2009;2. Art. N CD006142.

17. Cooper BC. The role of bioelectronic instruments in the management of TMD. NY State Dent J 1995;61(9):48-53.

18. Gomez CE, Christensen LV. Stimulus-response latencies of two instruments delivering transcutaneous electrical neuromuscular stimulation (TENS). J Oral Rehabil 1991;18(1): 87-94.

19. Melzack R, Wall PD. Pain mechanisms: a new theory. Sci 1965;150:971-979.

20. Núñez SC, Garcez AS, Suzuki SS, et al. Management of mouth opening in patients with Temporomandibular disorders through Low-Level laser therapy and transcutaneous electrical neural stimulation. Photomed Laser Surg 2006;24(1):45-49.

21. Didier H, Marchetti C, Borromeo G, et al. Persistent idiopathic facial pain: multidisciplinary approach and assumption of comorbidity. Neurol Sci 2010;31(Suppl 1):189-195.

22. Alvarez-Arenal A, Junquera LM, Fernández JP, et al. Effect of occlusal splint and transcutaneous electric nerve stimulation on the signs and symptoms of temporomandibular disorders in patients with bruxism. J Oral Rehabil 2002;29(9):858-863.

23. Allen JD, Rivera-Morales WC, Zwemer JD. Occurrence of temporomandibular disorder symptoms in healthy young adults with and without evidence of bruxism. Cranio 1990; 8(4):312-318.

24. Sheikholeslam A, Holmgren K, Riise C. Therapeutic effects of the plane occlusal splint on signs and symptoms of craniomandibular disorders in patients with nocturnal bruxism. J Oral Rehabil 1993;20(5):473-482. 